\title{
Civilisations
}

Revue internationale d'anthropologie et de sciences

humaines

58-1 | 2009

American Afrocentrism(e)s américains

\section{Afrocentrisme et littérature}

Le cas de l'écrivain noir américain John Edgar Wideman

Bénédicte Monville de Cecco

\section{(2) OpenEdition}

\section{Journals}

Édition électronique

URL : http://journals.openedition.org/civilisations/1909

DOI : 10.4000/civilisations. 1909

ISSN : 2032-0442

Éditeur

Institut de sociologie de l'Université Libre de Bruxelles

\section{Édition imprimée}

Date de publication : 31 août 2009

Pagination : 55-72

ISBN : 2-87263-026-0

ISSN : 0009-8140

Référence électronique

Bénédicte Monville de Cecco, « Afrocentrisme et littérature », Civilisations [En ligne], 58-1 | 2009, mis en ligne le 31 août 2012, consulté le 01 mai 2019. URL : http://journals.openedition.org/civilisations/1909 ; DOI : 10.4000/civilisations.1909 


\title{
Afrocentrisme et littérature \\ Le cas de l'écrivain noir américain John Edgar Wideman
}

\author{
Bénédicte MONVILLE DE CECCO
}

Résumé : Cet article s'intéresse à la production de discours critiques aux Etats-Unis qui, à propos de l'écrivain noir américain John Edgar Wideman et de sa littérature, mobilisent certaines des catégories théoriques de l'Afrocentrisme. Il y est question de la manière dont ces critiques, littéraires et académiques, contribuent à produire ce qu'elles se donnent pour objectif de décrire, c'est-à-dire un écrivain " afrocentré 》 ou simplement " africain américain ». Ce faisant, elles échouent, le plus souvent, à cerner la complexité d'une figure d'écrivain qui remet en cause de telles catégories, et ignorent que la trajectoire sociale et la reconnaissance de John E. Wideman éclairent les processus de consécration et d'autonomisation du champ littéraire.

Mots-clés : Afrocentrisme, critique littéraire, John Edgar Wideman, Etats-Unis, champ littéraire.

\begin{abstract}
This paper discusses the production of critical discourses in the United States which analyze the figure of the black American writer John Edgar Wideman through the lens of theoretical categories of Afrocentrism. The article shows that these discourses actually produce what they aim to describe, that is an "afrocentered" or simply an "African American" writer. However, these literary critics fail to figure out the complexity of a writer which precisely questions afrocentric categories. Moreover, such discourses ignore that Wideman's social trajectory and literary recognition highlight processes of cultural legitimization and of the construction of the autonomy of the literary field.
\end{abstract}

Key words: Afrocentrism, Literary criticism, John Edgar Wideman, United States of America, Literary field. 
L'Afrocentricité nous presse, nous commande de nous réinscrire, de nous repenser comme sujets de notre propre existence et d'en tirer, de façon systématique, toutes les implications. Il s'agit-là d'une démarche profondément révolutionnaire qui assène un coup fatal à la prétention et à l'arrogance occidentales dans la mesure où elle n'exige rien de moins qu'une rupture épistémologique d'avec l'Occident et une reconstruction volontaire et consciente de nous-mêmes sur des bases africaines.

(Ama Mazama, «Introduction à la traduction française », in Molefi Kete Asante 2003 : 5)

... to become a writer committed to telling the truth about color and oppression, a writer who exposes the lies of race and reveals how the concept of race is used as a weapon to destroy people.

(John Edgar Wideman $2008: 4$ )

L

dynamisme de la littérature de fiction nord-américaine qui s'affirme comme un des épicentres de la modernité littéraire, l'existence d'une figure d'écrivain noir constituée et sa relation complexe aux champs littéraires, national et international, la trajectoire sociale de l'écrivain noir John Edgar Wideman, sa reconnaissance et sa lucidité en font une personnalité d'écrivain hors du commun. La réflexion sur la vie et l'œuvre de cet auteur semble être un point de départ idéal pour définir et cerner les relations qu'entretiennent Afrocentrisme et littérature aux Etats-Unis.

En effet, l'exigence afrocentriste place l'Afrique et les cultures humaines qui la composent au centre de toute démarche artistique ou intellectuelle ${ }^{1}$. L'Afrique sera donc la référence essentielle des discours que j'analyserai. Car l'écrivain John Edgar Wideman et sa littérature participent à la structuration de pratiques sociales dans le cadre desquelles les individus mobilisent l'Afrocentrisme comme une catégorie possible d'entendement. Sans se déclarer forcément " afrocentrée $»^{2}$, la critique littéraire de l'œuvre de John Edgar Wideman révèle, souvent, l'acculturation de ceux qui parlent ou écrivent aux principes de l'Afrocentrisme. L'analyse anthropologique de ces discours et de la démarche de l'écrivain renvoie aux schémas cognitifs et aux canevas discursifs qui concourent à la production sociale de l'Afrocentrisme aux Etats-Unis. Elle interroge les conditions de l'institutionnalisation d'une figure d'écrivain « africain-américain », celles de la formation et de la reproduction d'une orthodoxie littéraire, et celles du privilège épistémologique de ses promoteurs.

1 Cf. Encyclopedia of Black Studies, en particulier la voix : « The Afrocentric Idea » (Asante et Mazama (éds), 2005).

2 Une démarche critique résolument afrocentrée répond aux impératifs théoriques suivants : « Afrocentric criticism is an evaluative field that uses the position of African agency to determinate the location of a text or situation (...). There are two dimensions to consider in determining centrality in a texte : (1) the writing, that is written about, and (2) the writer, who is doing the writing. It is only in capturing both the writing and the writer that Afrocentruc criticism can discover the total centrality of a text » (Asante et Mazama (éds) 2005: 62). 


\section{"The Astonishing John Edgar Wideman "}

John Edgar Wideman est né en 1941 à Washington D.C. Il grandit d'abord à Homewood, ghetto noir de Pittsburgh en Pennsylvanie, où sa famille est installée depuis plusieurs générations. Il a dix ans quand ses parents déménagent à Shadyside, un quartier blanc, dans l'espoir de garantir à leurs enfants une meilleure éducation scolaire. Wideman y entre au lycée où il excelle scolairement et devient capitaine de l'équipe de basket.

En 1959, il obtient le Benjamin Franklin Scholarship qui lui permet d'intégrer l'université de Pennsylvanie à Philadelphie; il est l'un des six étudiants noirs du campus, me dira-t-il. Suivent une série de distinctions académiques prestigieuses jusqu'à l'obtention, en 1963, du Rhodes Scholarship, grâce auquel il part étudier deux ans à Oxford en Angleterre. La même année, Look Magazine consacre un article au brillant étudiant et remarquable joueur de basket; "The Astonishing John Edgar Wideman ». Il a 22 ans et déclare qu'être noir n'a encore jamais constitué pour lui un réel handicap ${ }^{3}$. Trois ans plus tard, en 1966, il est admis à participer au prestigieux Iowa's Writers' Workshop et termine son premier roman.

En 1983, John Edgar Wideman reçoit le PEN/Faulkner Award pour son livre Le rocking-chair qui bat la mesure. En 1984, son essai Suis-je le gardien de mon frère? est nominé pour le National Book Award. En 1990, son roman L'incendie de Philadelphie 4 est distingué par un second PEN/Faulkner Award. C'est la première fois dans l'histoire du prix qu'un écrivain est primé deux fois. En 1993, John E. Wideman est récompensé par le MacArthur " Genius » Fellowship. En 1995, son livre Fatheralong : A Meditation on Fathers and Sons, Race and Society est à son tour nominé pour le National Book Award. La liste n'est pas exhaustive.

En fait, la carrière de John Edgar Wideman impressionne par la fulgurance et l'exceptionnalité de sa réussite. Il compte parmi les écrivains américains contemporains les plus importants.

\section{Le thème de la conversion}

Les critiques s'accordent pour distinguer dans la carrière littéraire de Wideman deux moments ${ }^{5}$. Le premier correspondrait à la publication de ses trois premiers romans et à la production d'une littérature « eurocentrée » par un écrivain en état de sujétion intellectuelle. Le second, à la production d'œuvres marquées par une démarche artistique plus « afrocentrée », par un écrivain affranchi.

3 « If there were something I wanted very badly that being Negro prevented me from doing, the, I might have the confrontation of a racial problem, and I would be driven to do something about it. I'm sure I would. But so far, the things that I've wanted to do haven't been held back from me because of my being a Negro. So the problem is not my own problem, not something I feel I have to cope with or resolve » (Wideman, in Shalit 1998 [1963]: 2).

4 Son oeuvre est partiellement traduite en français et publiée aux éditions Jacques Bertoin et Gallimard. J'ai choisi, quand c'était possible, de citer les titres français. John Edgar Wideman est également l'éditeur de plusieurs volumes importants de littérature dont My Soul has grown deep, Classic of Early AfricanAmerican Literature, New York, The Ballantine Publishing Group, 2002.

5 D'une manière générale, ce commentaire est partagé par l'ensemble de la critique littéraire et académique. Pour plus de précision, on peut se reporter aux travaux de Keith E. Byerman (2006), James Coleman (1989), Raymond E. Janifer (1997), Dorothea D. Mbalia (1995) et Bonnie TuSmith (1998 et 2006). 
Son premier roman A Glance Away (1967) vaut à Wideman ses titres de noblesse littéraire. C'est la comparaison avec des écrivains modernes irlandais et anglophones et la capacité de l'écrivain à sortir des supposés poncifs qui caractériseraient la littérature de ses pairs, écrivains noirs, qui servent au critique du New York Times à nous assurer de son talent.

\begin{abstract}
He [John Edgar Wideman] has all sorts of literary gifts, including a poet's flair for a taut, meaningful, emotional language. With this book, another young Negro writer has established himself in a vein of contemporary American fiction; another craftsman has learned, from Irishmen like Liam O'Flaherty and James Joyce, what makes the world of words and the interaction of dreams reach the sensual state of literature. (...) This book, however, develops an enormous objectivity in it characterizations. Mr. Wideman has written a powerfully inventive novel (Roskolenko ${ }^{6}$, le 10 septembre 1967).
\end{abstract}

En 1970, il publie son deuxième roman Hurry Home et, dans le même journal, un autre écrivain-critique reproche à Wideman de donner du ghetto noir américain une vision dépassée et conciliante.

While many of the scenes take place in the urban ghetto of the mid-sixties, the ambiance is that of an earlier, less volatile decade. Little or no mention is made of such timely realities as mass violence, and the protagonist, an educated and articulate man, is chary of all but the most conciliatory politics (Joseph Goodman, le 19 avril 1970).

Keith E. Byerman, professeur à Indiana State University et spécialiste de littérature africaine américaine, considère que ces deux premiers romans ont été l'objet d'une attention critique relativement faible « parce que leur engagement moderniste manifeste les place hors des modèles conventionnels de la littérature africaine américaine ${ }^{7}$. En 1973, The Lynchers conclut la première phase de la carrière littéraire de Wideman. Pour Raymond E. Janifer, professeur d'anglais à l'Université de Shippensburg, c'est un roman de transition. Un point de vue partagé par James Coleman qui remarque, dans l'essai qu'il consacre à l'auteur, que malgré l'importance croissante de la « tradition historique et culturelle et du langage noirs » dans ce roman, Wideman ne parvient pas encore à cette voix noire pleine et mature qui caractérisera sa Trilogie de Homewood ${ }^{8}$ (1989: 44 et 153).

Cette représentation de la carrière littéraire de John Edgar Wideman avec son moment central, marqué par huit années de silence, est partagée par l'ensemble de la critique. Elle dessine l'image récurrente de la conversion de l'écrivain à un art plus authentiquement

6 Journaliste, écrivain et poète, né en 1907 de parents juifs nés russes, et mort en 1980. Roskolenko écrit pour The New York Time Book Review à partir de 1944. Pendant la première guerre mondiale, il séjourne en Australie. Il entretiendra, toute sa vie, une relation intellectuelle et affective particulière avec ce pays.

7 « John Edgar Wideman's first two novels, A Glance Away (1967) and Hurry Home (1970), have received relatively little critical attention, in large part, I would argue, because the overt engagement with high modernism places them outside the conventional model of African American Writing » (Keith E. Byerman $2006: 93)$.

8 La critique désigne communément sous le nom de Trilogie de Homewood les trois romans que Wideman a consacré à l'histoire de ce quartier éponyme de Pittsburgh où il a en partie grandi et où sa famille est implantée depuis plusieurs générations : Damballah, Où se cacher et Le rocking-chair qui bat la mesure, publié respectivement aux Etats-Unis sous les titres Damballah, Hiding Place et Sent for You Yesterday, en 1981, 1981 et 1983 et, en France, en 2004, 2006 et 2008 aux éditions Gallimard. 
« africain américain » (Byerman 2006 : XII). Représentation confortée par le discours de l'écrivain lui-même qui reconnaît avoir modifié sa manière d'écrire et s'être davantage inspiré de sa culture d'origine. «By 1973 I'd published three critically acclaimed novels. It was hard to admit to myself that I'd just begun learning how to write, that whole regions of my experience, the core of the language and culture that nurtured me had been barely touched by my writing up to that point » (Wideman, cité par Bonetti 1998 [1985] : 47).

Pourtant, si ce dernier sacrifie partiellement au motif de la rupture, il souligne la cohérence d'un parcours qui ne peut être compris que dans sa continuité. "One way they differ is simple enough, and that is I get older, and as I get older, maybe I become exposed to more. And I think also I grow. ... I hope I am growing more independent » (in Coleman 1989 : 148).

Or, Dorothéa Mbalia, critique résolument afrocentriste, envisage ce changement d'état, d'un écrivain dominé par « l'histoire, la culture et le langage de l'Europe » à un écrivain qui « accepte et apprécie l'histoire, la culture et le langage africain », comme une conversion radicale. La culture académique de Wideman aurait été éradiquée et remplacée par sa culture africaine, la seule qui compte vraiment, et qui lui aurait enfin été révélée.

In his acceptance of his past mistakes, in his struggle to come up with answers that will prevent future fires that originate from Africans' own self-hatred (...) Wideman redeems himself.

In many ways then, Philadelphia Fire is about Wideman's own internal fire, a fire that completely burns out the old European-centered perspective and gives birth to a new African-centered one. It is a baptism of fire that whips the intellectual Wideman home, that drives him to reclaim his African personality, just as it drives Cudjoe home: Back Again, Black Again. Home (Mbalia 1995 : 97).

Ce discours, pétri de religiosité ( redeem », " baptism »), témoigne une vision manichéenne de l'évolution de l'écrivain cohérente avec la théorie afrocentriste?. L'intellectuel « africain » sera, en dernière instance et quelque soit son degré d'aliénation, touché par la grâce de l'afrocentricité et échappera au destin identitaire minoritaire qui lui était réservé.

Although his early works are disapointingly Eurocentric, his later works document his efforts to reclaim the African Personality. These later works, the stories of John Edgar Wideman in particular, reflect Wideman's awareness that no matter where African people live throughout the world, they share a common oppression and a common history. These later works also demonstrate that change does indeed occur, that African people no matter how far outside the fold they may have wandered, can return and redeem themselves by servicing African people and their communities (Mbalia 1995 : 9).

Marginale dans sa forme totalitaire, cette interprétation afrocentriste sous-tend cependant le sens de nombreuses critiques qui insistent sur l'évolution individuelle de l'écrivain et conçoivent des systèmes culturels clos et incompatibles. "Wideman embracing Afrocentrism and combining it with the folklore of the traditional AfricanAmerican ethnic culture afforted him the opportunity to resolve his 'psychic duality'

9 « L'Afrocentricité ne vous convertit pas en faisant appel à la haine ou à la convoitise, à la rapacité ou à la violence. En tant qu'idéologie consciente la plus élevée, elle affirme ses principes, motive ses adhérents, et gagne les prudents par la force de sa vérité » (Asante $2003: 19$ ). 
complex » (Janifer $1997:$ 15). Ce faisant, elles ignorent les composantes structurellesclés de son expérience de vie, négligent les processus sociaux d'inscription identitaire, et en particulier, selon les mots que Pierre Bourdieu mettait sur sa propre expérience, " l'effet durable d'un très fort décalage entre une haute consécration scolaire et une basse extraction sociale, c'est-à-dire l'habitus clivé » (Bourdieu 2004 : 127), à quoi s'ajoute ici une appartenance « raciale » stigmatisée. «I talked to you about my own life experiences, how so much was crammed in a very short period. That creates dislocations, crazy juxtapositions. One day at home, I have to stomp on a roach; next day, I am eating caviar and drinking Stolichnaya » (Wideman, in Samuels 1998 [1983]: 29).

Car la personnalité de cet écrivain ne saurait être envisagée comme l'expression de stratégies arbitraires dans la mesure où celles-ci, qu'elles soient le fait de l'écrivain ou de ceux qui le reçoivent, subissent la contrainte de formes culturelles existantes qui sont elles-mêmes le produit et le support de luttes sociales. Dans les termes de Frantz Fanon : « Et voilà, ce n'est pas moi qui me crée un sens, mais c'est le sens qui était là, pré-existant, m'attendant. Ce n'est pas avec ma misère de mauvais nègre, mes dents de mauvais nègre, ma faim de mauvais nègre, que je modèle le flambeau pour $\mathrm{y}$ foutre le feu afin d'incendier ce monde, mais c'est le flambeau qui était là, attendant cette chance historique » (Fanon 1952: 109).

\section{La constante africaine}

Les partisans de l'afrocentricité postulent un rapport d'identité entre les Noirs américains et les Africains. «(...) because African and of course Afro-American are close and at a lot of point not separable (...) » (Coleman 1989 : 153). Ils récusent l'existence d'une identité prescrite et dégradée qu'ils tentent de dépasser en en élargissant les contours de manière à y inclure d'autres Noirs et en particulier des Africains ${ }^{10}$. Ils requalifient les Noirs américains en «Africains » ou en «Africains américains » et entendent témoigner de l'identité ethnique (plus rarement raciale ${ }^{11}$ ) des Américains noirs sur la base d'une identité africaine postulée. Or cette idée que « les Africains américains sont un groupe ethnique africain dont les membres sont citoyens des Etats-Unis d'Amérique » (Asante et Mazama $2005: 8$ ), qui a pu constituer une réponse politique forte aux tentatives d'isolement et de relégation, sociale et politique, de la population noire aux Etats-Unis, n'est possible qu'au prix d'un révisionnisme historique qui nie le rôle social déterminant de l'institution esclavagiste. " Les Noirs sont incités, si ce n'est à oublier l'expérience de l'esclavage qui apparaît comme une aberration dans le récit grandiose de l'histoire africaine, du moins à replacer celle-ci au centre de leur réflexion et à l'associer à une idée mystique et implacablement positive de l'Afrique (...)» (Gilroy 2003 : 250). En effet, l'Afrocentrisme considère l'esclavage comme « le lieu de la victimisation des Noirs, et donc l'effacement

10 « Racist reaction in the period of trauma forced blacks into self-defensive, locally based communities; through their representations intellectuals and others leaders sought to broaden this to include other blacks or, for some, Africans and all people of color» (Eyerman $2001: 59)$.

11 «Les Africains américains constituent le second plus grand groupe racial aux Etats-Unis d'Amérique » (je traduis, Asante et Mazama éds. $2005: 8$ ). 
programmé de la tradition » (Gilroy 2003 : 249) et l'expression " slave mentality » ${ }^{12}$ que la critique Dorothea Mbalia emploie plusieurs fois pour qualifier l'écrivain Wideman des premières œuvres est significative d'une vision de l'esclave comme d'un être totalement aliéné au modèle dominant contradictoire avec l'historiographie de l'esclavage ${ }^{13}$.

Dans Où se cacher, Tommy, sorte de double fictionnel du plus jeune frère de Wideman, pense en observant la cahute où vit Bess, son arrière grand-tante : " C'est tout ce qu'y a hormis le fourneau et les étagères le long des murs, à peu près comme si elle vivait oubliée au fin fond de nulle part où les nègres vivent encore comme des Africains ou des esclaves » (2006 [1981] : 112). Ces trois figures nettement distinguées du nègre, de l'esclave et de l'Africain ne recoupent en aucune manière celles convoquées par Asante, théoricien de l'afrocentricité ; «Ainsi, si l'on veut se concentrer sur l'étude des Africains des cités du Nord-Est des Etats-Unis, ce qui est raisonnable, il faut le faire en gardant à l'esprit l'idée que les gens qu'on étudie sont des Africains, pas des « nègres made in America » sans profondeur historique » (Asante, cité par Gilroy 2003 : 250). Bess, quant à elle, déclare ; «On laisse tomber les chiffres pairs. On les donne aux Blancs. Ils aiment quand deux et deux font quatre. Deux-quatre-six-huit-dix. Deux par deux, quatre à quatre et dix petits Indiens en file indienne. Ils aiment les trucs qui ont des angles, les trucs qu'on peut casser en deux, les trucs ayant une nature qu'on peut dompter » (2006 [1981] : 206). Réminiscences ou non du texte d'Aimé Césaire Cahier d'un retour au pays natal : «Parce que nous vous haïssons vous et votre raison, nous nous réclamons de la démence précoce de la folie flambante du cannibalisme tenace (...)/Et vous savez le reste/Que 2 et 2 font 5 (...) » (1994:26). Chez Wideman, l'acte d'énonciation a un effet performatif immédiat et l'écrivain actualise les représentations contrastées de deux raisons opposées l'une à l'autre. Cette revendication ironique d'une identité prescrite, en l'occurrence celle du Noir comme d'un individu dissipé et irrationnel incapable de prendre part au

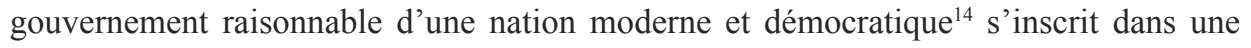
tradition intellectuelle qui entend, dans les années 1930-1950, " ébranler » les assises symboliques d'un pouvoir raciste en inversant la valeur de ses représentations. Il ne s'agit pas de retrouver une africanité perdue mais de dénoncer l'arbitraire et la fonctionnalité d'images racistes qui pèsent sur le devenir du Noir américain. Car la racialisation de l'identité noire aux Etats-Unis apparaît historiquement comme la justification ultime à un ordre social esclavagiste et post-esclavagiste où des couleurs de peau sont réduites à une

12 «That is, he sees himself, his culture, his people through the eyes of a European. Such a distorted perspective, an anti-self perspective, makes him view writing as an act of superiority; forces him to see his audience as a European one; makes him choose « beat generation » themes (such as the Oedipus complex, the world as a wasteland, the intellectual's alienation from society); allows him to reject most things associated with African people; and forces him to write according to the literary standards of the West. By doing so, Wideman reveals his acceptance of slave status and reflects the slave mentality of the African who experiences a personality crisis » (Mbalia $1995: 34)$.

13 Cf. Elizabeth Fox-Genovese 1988, le chapitre 6 « Women Who Opposed Slavery »; Darlene C. Hine, 1979 (3) : 123-127; John Hope Franklin \& Alfred A. Moss 2005, le chapitre 8 « That Peculiar Institution » et Ellen Ginzburg Migliorino 1994, le premier chapitre.

14 Cette vision du Noir, dissipé et irrationnel, s'impose après l'abolition de l'esclavage. Elle permet, au moment de l'instauration d'un régime légal de ségrégation, d'imputer aux Noirs eux-mêmes la responsabilité de leur irresponsabilité civile ; "African Americans were viewed as aliens whose ignorance, poverty, and racial inferiority were incompatible with logical and orderly processes of government » (Franklin \& Moss 2005 : 288-289). 
couleur de peau commuée, assez vite, en une identité « noire ». Laquelle, réifiée en une série de préjugés racistes, constitue l'explication ultime au bien fondé de l'exploitation économique et de l'aliénation politique de la population noire américaine et de la suprématie blanche. «Les esclaves deviennent une masse anonyme dissipée, non fiable et paresseuse. Ils s'opposent au « freeman » libre politiquement et indépendant économiquement qu'ils contribuent à définir » (Wacquant 2005 : 135). Après la guerre civile, cette représentation est réactualisée par la réaction raciste qui est l'occasion pour la nation américaine de se réunir en une nouvelle narration où les Noirs en général deviennent des objets de haine, ceux contre lesquels les deux bords peuvent s'unir et se réconcilier.

Dans ce contexte, Wideman ne postule aucune identité ethnique entre les Noirs américains et les Africains mais des analogies structurelles et un lien historique qui privilégient, par exemple, la comparaison du destin des jeunes hommes noirs aux EtatsUnis avec celui des Xhosas d'Afrique du Sud.

\section{Abattu. Abattoir. Les jeunes Noirs s'abattent entre eux. Se tuent. Abattus. Abattoir. \\ (...) \\ Le bétail est le peuple. Le peuple est le bétail. \\ Chanson d'amour dans le temps. Puis chant funèbre. Cette image le hante. Le peuple xhosa tuant son bétail, se tuant, un monde qui se défait (1998 [1996] : 18).}

Car, son œuvre multiplie les références aux sociétés et aux cultures africaines qui deviennent des métaphores possibles du monde américain contemporain. En effet, l'écrivain déclare travailler à la reconstruction du sens de la réalité ; "And Reuben himself, the charactère within the novel, is engaged in a struggle that I think I'm engaged in as a writer outside the novel, creating and sustaining a version of reality to compete with those destructive versions which are tearing us apart " (Wideman, in Coleman 1989 : 152). Partant, il modifie les références à l'aune desquelles nous construisons les représentations qui tiennent l'ordre en place; "You can understand the American experience or the AfroAmerican experience in Kongo terms as well as in terms of Calvinism and Protestantism and Roman Catholicism and Western humanism. Those are ways, those are containersjails for culture-which don't explain everything that needs to be explained, so I'm looking for other explanations » (Wideman, in Coleman 1989 : 154).

Wideman remplace momentanément un système de référence par un autre en livrant, par exemple, les réflexions d'un Africain arraché à sa terre, il témoigne de la relativité des points de vue et du caractère culturel des perceptions et des représentations.

Nous avions entendu chez nous, en Afrique, avant que d'avoir été kidnappés, parler de cannibales qui vivaient de l'autre côté de la grande eau. Le premier jour que je passai à la tannerie, abasourdi par le vacarme, la puanteur, les fleuves de sang, j'eus peur d'être tombé à l'endroit même où les mangeurs d'hommes au visage rose préparaient leur monstrueux festin (1998 [1996] : 137).

Mais nous sommes ici très loin d'une critique afrocentriste dogmatique qui érige les catégories esthétiques édifiées par les individus en systèmes antagonistes et exclusifs : les personnages de Wideman ne sont jamais parfaitement dupes du caractère politique et culturel de ces catégories. Albert Wilkes, saisi par la beauté d'une femme blanche qu'il retrouve après sept ans d'absence, se souvient de ce père brutal qui le renvoyait à sa condition de « nègre ». 
Il se rappelle les seuls mots prononcés par son père et restés dans son souvenir : Ta tête de noir est pas faite pour un oreiller de satin. Baffe à l'appui, qu'il en voit trente-six chandelles (...) N'empêche que du satin, il en avait des kilomètres carrés, là, sous les yeux, et bel et bien du satin sous sa tête de noir lorsque sa julie se refaisait toute belle, toute douce, toute parfumée dans une salle d'eau plus grande que toute la cabane qu'ébranlait de sa grosse voix son papa. Une belle julie plus blanche que les rêves les plus blancs de son papa. Blanche à en pouvoir être noire si elle le désirait. Parler comme les nègres, se conduire comme les nègres. Traiter les blancs comme les nègres aimeraient le faire. Etre noire quand ils jouent sous ces draps de satin. (...) (2008 [1983] : 97-98).

Le lecteur comprend, bien que le rapport ne soit jamais explicitement énoncé, que le bonheur sincère d'Albert Wilkes à aimer une femme blanche est au moins partiellement lié à la revanche qu'il prend sur le destin que lui promettait sa condition sociale dont la violence symbolique était incarnée par la violence paternelle. Il sait que, dans cette société raciste, les processus de prescription identitaire conditionnent des attitudes de " nègre ", qu'on « parle comme un nègre ", qu'on " bouge comme un nègre », qu'on devient « nègre » dans un devenir " nègre » de la société toute entière et qu'il n'est pas possible à ceux qui ont la peau noire de s'y soustraire totalement.

Aussi, l'interprétation critique afrocentriste limite considérablement la portée anthropologique de la démarche de l'écrivain quand elle se cantonne à remarquer qu'en introduisant des éléments de culture orale, c'est-à-dire en empruntant des motifs culturels à un monde noir américain considéré, à tort ou à raison, comme un monde oral, l'écrivain reconnaît la proximité entre les Noirs américains et les Africains ; "Samuels : May. Again, there is a character like her in your short stories. And the one thing that stuck me about her is that she is a kind of griot, in the African sense: she is the story teller, the family historian who knows the lore of the family » (Samuels 1998 [1983] :14). Elle ignore les tensions et les conjonctions qui caractérisent les situations d'interface entre l'oral et l'écrit ${ }^{15}$. Le retour à contresens de l'écrivain sur la ligne horizontale d'une évolution linéaire qui comprend le rapport entre l'oral et l'écrit comme un rapport hiérarchique, qui n'est autre que la projection vulgaire de l'opposition entre raison graphique et raison orale, déconstruit le rapport hiérarchique dont on peut identifier l'empreinte à tous les niveaux de la société ; de la relation de l'esclave au maître, à celle, aujourd'hui, de l'illettré marginalisé au lettré intégré ${ }^{16}$. Elle méconnaît que, ce faisant, l'écrivain questionne les représentations qui soutiennent et naturalisent les rapports de domination et elle occulte la dimension strictement sociale des mécanismes culturels de domination qui inhibent les membres des classes populaires en imposant une idée très conservatrice de ce qu'il est ou n'est pas possible d'écrire.

Wideman: (...) So I started with a very conservative idea about what was okay to reveal and what wasn't okay to reveal, combined with the powerful cultural imperative that you don't tell most of what you know. It would get you in trouble.

Interviewer: Baldwin writes about that.

Wideman: It's crucial. You can find the same proverbs among Russian cerfs.

Interviewer: It's the underclass, whatever that class is.

15 Cf. Jack Goody 1979 et le numéro que lui consacre la revue Pratiques : (131-132) « La littératie. Autour de Jack Goody », décembre 2006. 
Wideman: Exactly. You can't speak what's on your mind, you can't be frank with people because it will be used against you. Your language and your customs, etc. revolve around the hard, cold facts of your servitude, of the oppressed state that you live in (Bonetti 1985 : 54).

Certes, l'esthétique « afrocentrée » construit une subjectivité qui permet à l'individu noir d'échapper à la honte à laquelle il est socialement voué sans pour autant tomber dans les travers de la haine de soi. Mais l'Afrocentrisme remplace souvent, des savoirs constitués et des pouvoirs établis par de nouveaux savoirs et pouvoirs qui entendent l'identité noire non plus relativement à l'étalon homme-blanc-adulte-libre-middleclass-hétérosexuel mais à une nouvelle constante, " africaine » cette fois. Ce faisant, il substitue au sujet racisé humilié un sujet racisé et fier de lui-même. En déniant le caractère relationnel des identités qu'il réifie, il échoue à sortir des modèles essentialistes de l'identité.

\section{Wideman : un écrivain africain-américain ?}

Les éditeurs du dernier recueil d'essais consacré à l'écrivain, publié en 2006 et seul de cette envergure, notent en quatrième de couverture que " malgré des perspectives critiques diverses, les contributeurs placent Wideman au centre de la littérature africaine américaine en tant qu'exemple des approches postmodernes en littérature » (je traduis, in TuSmith et Byerman (éds) 2006). On peine à comprendre ce que signifie cette proposition si on n'interroge pas le rapport qu'entretiennent « la littérature africaine américaine » et « la littérature », distinguées et instituées par le discours de la critique littéraire.

En effet, à l'instar de Dorothea Mbalia ${ }^{17}$, Raymond E. Janifer ou James W. Coleman, de nombreux critiques affirment l'existence d'une littérature " africaine américaine » avec ses propres standards. De fait, les écrivains noirs sont systématiquement renvoyés à des catégories spécifiques, des étagères des librairies "African American Fiction », aux anthologies, "African American Literature », en passant par les départements des universités où leurs œuvres sont enseignées, "African American Studies ». Pourtant, la trajectoire de John Edgar Wideman, aux confins des champs littéraires « africain américain » et « américain », relativise la vision afrocentriste d'un champ littéraire africain américain autonome. En avril 2005, lors d'un entretien qu'il m'accordait à New York au moment où sa nouvelle consécration internationale stabilisait sa position nationale, Wideman me déclarait :

Here is the funny problem for a person like myself. For people who are organizing these little shelves, I'm somebody in between. I'm not black enough for the black shelves and too black for the white shelves, so " ain't no shelves! "It's very discouraging in that sense: too experimental for the traditional, too traditional for the experimental. I mean you just begin to wipe out whole careers, you begin to wipe out the people who are attempting to work "between " who are attempting to merge genre as a merge category, broaden a kind of political or aesthetic frame in which these categories exist. But that's ok. That's the way it has always been in the world of literature.

If you wanna do something different you're gonna bump into people who don't want to hear something different, read something different. I don't know how you solve that problem (Wideman, in Monville De Cecco 2005).

17 «Of course this evolution also impacts upon the writing process; it frees him from the constraints of western literary tradition and centers him within the African tradition, a tradition which has its own standards that are always being creatively renamed and revised » (1995: 28). 
Ici, la théorie des champs et la notion d'habitus clivé élaborée par Pierre Bourdieu s'avèrent d'une grande utilité pour appréhender les relations qui, au sein de la société globale et du champ littéraire en particulier, rendent intelligible son expérience spécifique. En fait, l'écrivain qui ambitionne de devenir un « world class writer » doit, pour atteindre le sommet de la hiérarchie littéraire, satisfaire aux canons littéraires dominants.
All of us grow up very confused and I thought writing was something that was connected with Europe. The matter of Europe. I didn't want to be a good American writer, let alone a good black writer. I wanted to be world class, man, and to be world class you had to be Thomas Mann and you had to be Marcel Proust and you had to walk along the Champs Elysées and you had to know bullfights (Wideman, in Bonetti 1998 [1985] : 44).

Pourtant, si l'acculturation de l'écrivain Wideman, qui avait préalablement du se plier aux exigences des systèmes scolaires et universitaires, fut la condition nécessaire pour son incorporation au courant dominant du champ littéraire, elle ne fut cependant pas suffisante à assurer sa visibilité nationale.

To write the very best, didn't you have to cheat a little, didn't you have to "transcend Blackness "? Didn't you have to ground yourself in an experience that was outside... Homewood? Didn't you have to show you were part of a larger world? Didn't you have to show continually your credentials with allusions to the "great writers »-the great traditions? (Wideman, in Samuels 1998 [1983] : 17).

C'est que la position spécifique de l'écrivain en fait une figure possible de l'autonomisation du champ littéraire. En effet, aux dispositions déterminées par l'appartenance sociale se conjuguent des dispositions déterminées par l'appartenance à une catégorie « raciale » stigmatisée qui, une fois constituée et à condition que perdurent les circonstances politiques qui en assurent la reproduction, en raison de la nature même du seul critère objectif ${ }^{18}$ à partir duquel elle se constitue, la couleur de la peau, devient indépassable ${ }^{19}$. «L'identité noire », capable de rassembler des hommes que leurs différences sociales et statutaires distingueraient fortement, pose, dès lors, le problème de la complexité de la construction des habitus. "L'injure » (au sens où la définit Didier Eribon) permanente que représente le racisme de la société américaine est « constitutive de la subjectivité des individus stigmatisés »(Eribon 1999). Ainsi, l'écrivain déclare ressentir l'inconfort d'une position privilégiée au sein de l'académie, marquée par la précarité de sa position au sein de la société toute entière : « Of course I felt privileged to have a job there [The University of Pensylvannia] and lucky to be in a position to perkage of the bounty, but then again a destructive kind of guilt came along with the goodies. I was a black face in a white sea (...)» (Wideman, in Rowell 1998 [1989] : 91) ; « Que je

18 Des études de cas spécifiques témoignent de la subjectivité des perceptions et montrent que la couleur de la peau relève, en partie, d'une construction mentale. Ce qu'expriment également les perceptions étonnement variées de la couleur de peau du président Barack Obama et qui relativise l'objectivité que je postule ici. Voir aussi les travaux de Jean-Luc Bonniol sur les Antilles françaises 1992 et 2001.

19 « C'est peut-être trop peu reconnaître, même pour les besoins de la thèse, combien les Américains noirs peuvent être américains et combien sont également très américaines les prétendues solutions d'un problème qui est fondamentalement une question d'oppression économique et de racisme. Qu'il existe ou non une culture noire, la spécificité du cas afro-américain réside dans la particulière oppression - souvent et presque partout exercée - que rend possible la différence de couleur » (Mintz 1971 : 118). 
sois écrivain et professeur de littérature à l'université, je suis Noir. Robby est mon frère. Ces faits m'incrimineraient toujours » (Wideman 1992 [1984] : 27).

Il devient un insider/outsider ${ }^{20}$, comme il se définit lui-même ${ }^{21}$, ce qui rend problématique son acculturation totale aux valeurs dominantes des champs du pouvoir politique et du pouvoir économique et le place d'emblée dans une situation favorable face au champ artistique. Car, aux Etats-Unis, la sincérité de l'engagement philanthropique de milliers de fondations, mécènes et universités qui dominent l'univers culturel est garantie par l'indépendance de leurs politiques artistiques à l'égard des pouvoirs politique et économique qui, par ailleurs, les ont instituées ${ }^{22}$. Partant, l'écrivain suscite les expectatives de la critique la plus autonome, le plus souvent des écrivains, dont les choix relèvent aussi de stratégies qui visent à assurer leur propre position et légitimité23. D'où, d'une part, cet empressement, que nous relevions en première partie, à exiger de Wideman, après la publication de son deuxième roman, une plus grande radicalité et, d'autre part, une certaine réticence à lui accorder une attention critique, qui tarda d'ailleurs à arriver ${ }^{24}$.

C'est ainsi que le jeune écrivain noir dominé, rompu aux canons littéraires dominants, gagne en prestige et en reconnaissance en transformant son vécu, qui lui vaut par ailleurs d'être humilié, en matière littéraire. D'où, l'effet d'autonomisation que la captation de son œuvre et de sa personnalité d'écrivain peut avoir pour le champ littéraire. Mais, cette

20 «I grew up in a very segregated country. I knew that there are two worlds. I knew that I lived in one of them. And I knew that my world didn't count very much to most Americans. I also understood that I can move back and forth between those worlds. But if I did it would always be a little bit dangerous, a little bit painful, troublesome -that's just the way I thought the world always would be- and I had no ambition to necessarily try to change it because it seemed like a force of nature » (Wideman, in Monville De Cecco 2005 : entretien non publié).

21 «I was both participating but also forced to take the posture of an observer because I didn't know what the hell was going on. It was best to keep quiet and sort of check it out before O exposed my game. The insider/ outsider nature of my experience made me a listener » (Wideman, in Samuels $1983: 25$ ).

22 Cet élan philanthrope, qui trouve sûrement son origine dans un protestantisme ascétique, est devenu indispensable dans une société où la philanthropie « accroît la position sociale, qu'elle donne accès à un réseau de pairs de haut niveau, (...) aide à réussir dans les affaires par les contacts qu'elle procure sources d'autant de possibilités d'échanges et de commerce » (Martel $2006: 303$ ).

23 «Wideman: (...) Criticism is never disinteresting and so one question asked about any piece of criticism is what job is it trying to do, who is it working for, who is it serving? And when you ask that, you very often find out that it's certainly not working in the interest of the book that is being talked about. It's working in the interests of the existing categories, working in the interests of the existing politics. So there is a real antagonism between creative work, a building antagonism between creative work and criticism. The fate of the « avant-garde » (en français) is a perfect example always and I think there is a real suspicion among most critics. Most critics and certainly teachers are suspicious of literature. Really.

Even in their kind of warm up to it they often are looking for « it's like this » or « it's like that » or « it's like the other thing ». And that kind of energy is spent before a piece is looked at on its own terms or an attempt is found to get its own terms.

So good criticism is as rare as good fiction... Rarer.

Monville: I've noticed that many of the critics here in America are also writers.

Wideman: Yes. So, how seriously can you take the whole reviewing mechanism in this country, when only two or three reviews count for writers' reputation » (Wideman/Monville De Cecco 2005 : entretien non publié).

24 « In Wideman's case, it took an eight-year hiatus and the release of brothers and keepers (his first book with Holt) for him to achieve the type of media attention that sells books in a big way » (J. Rosen 1998 [1989] : 84). 
dynamique n'opère que dans la mesure où l'écrivain accepte momentanément l'autorité de formes reconnues. Aussi, la démarche artistique de Wideman n'entretient pas un rapport antagonique avec celle des écrivains intégrés au courant dominant du champ littéraire. Il existe au contraire un rapport de connivence dans la continuelle réinvention à la marge de formes littéraires qui reproduisent les conditions de l'autonomie du champ et assurent à leurs promoteurs une position centrale.

I felt that I had to prove something about black speech for instance, and about black culture, and that they needed to be imbedded within a larger frame. In other words, a quote from T.S. Eliot would authenticate a quote from my grand-mother. Or the quote from my grand-mother wasn't enouth, I had to have Joycean allusion to buttress it, to keep an awarness that " Hey, this is serious writing and this guy's not just a solitary black voice, but he knows the things that you know, he's part of the shared culture »(Wideman, in Bonetti $1985: 51$ ).

Autrement dit, il s'agit bien de témoigner, à l'instar de Raphaëlle Rérolle dans Le Monde, à la fois de la rupture et de la continuité d'une œuvre qui revitalise la littérature en remettant en cause les formes qui font autorité et qui, garanties par des autorités, en légitiment, dans un mouvement réciproque, l'autorité ; "Mieux qu'un épais bâton de dynamite, ses textes font voler en éclats les frontières d'une langue imposée par la culture dominante, celle des Blancs ; et cela en demeurant [de] magnifique[s] morceau[x] de littérature et de passion » (15 avril 2000).

En bousculant à la fois l'orthodoxie littéraire et l'autorité des " orthodoxes 》 de la littérature, Wideman travaille à la reproduction des conditions de l'autonomie du champ. La critique afrocentriste ne dit rien d'autre quand elle rapproche les techniques littéraires qu'il utilise de formes culturelles africaines tout en affirmant que la modernité de son œuvre réside justement dans sa capacité à miner les fondements de l'orthodoxie littéraire ${ }^{25}$. Pourtant, lorsqu'elle comprend son utilisation de la poly-focalisation comme une expression de son africanité retrouvée, elle trahit une vision stéréotypée de l'Afrique comme d'une entité indivisible et construit une autre chapelle ${ }^{26}$.

C'est bien la position particulière de John Edgar Wideman et des producteurs culturels noirs en général, face à ce que l'écrivain désigne comme le courant dominant aux EtatsUnis qui les met en situation de l'amender ${ }^{27}$. Cela ne signifie pas qu'ils transforment tous « l'essai ». D'autant que la force de la majorité réside dans sa capacité à intégrer, c'està-dire, à « naturaliser » ou, au contraire, à marginaliser ce qui, autrement, remettrait en cause son pouvoir symbolique. Lequel a pour enjeu « la formation » et la « ré-formation » des «structures mentales » (Bourdieu 1982). Si, la pratique de la littérature peut être considérée comme relevant d'une stratégie d'identification de la part de l'écrivain qui se

25 « Wideman's philosophy of time reflects the west African concept of Great Time (...) ) (H. R. Andrade $2006: 48)$.

26 Cf. Mbalia 1995: 16 et suivantes.

27 « The expressive arts produced by Black Americans are informed by rituals one of whose prime purposes is to transcend the limits and boundaries imposed by the power of the majority society. Bursting categories as basic as time and space, an alternate version of reality is formed as Black people live their lives outside of the " mainstream » of American culture. What Murray attests to is the power of these alternative versions, how they shatter the complacencies of a mainstream, now the notion of a placid, separate, abiding mainstream is a fiction once you go below the surface - whether of language or music - and ask a few of the right questions » (Wideman $1978: 30$ ). 
soustrait ainsi partiellement à la contradiction de la double prescription d'assimilation à l'identité dominante et d'assignation à une identité minoritaire, cette stratégie s'inscrit dans une histoire culturelle qui en détermine les limites. La constitution d'un champ littéraire « africain américain » avec ses propres instances de légitimation apparaît comme un moyen habile de préserver les catégories du statu quo.

There is an Afro-American, tradition. There are Afro-American writers working right now; It makes sense to talk about them as a group, or about certain ways that their work relates. It is natural; it is enlightening; it is intelligent to do it that way, but, at the same time, to do that perpetuates the "bad side " of the whole notion of looking at things in black and white ways (Wideman, Samuels 1998 [1983] : 22).

Dès lors, la position de John Edgar Wideman au sein du champ littéraire états-unien ne peut que raviver chez l'auteur la conscience de sa position d'Américain noir dominé dans la société globale et renforcer sa solidarité avec ses pairs. Tout comme elle irrigue une œuvre qui n'a de cesse d'interroger les mécanismes de la domination.

\begin{abstract}
But as I said before, the whole vexed relationship of Black literature to the mainstream, the inability of critics to treat Black literature as technique, as art, the tendency of critics to use Black literature as a way of trying to put forward certain ideological concerns and points of view-that has been a disaster for all Black writers (Wideman, in Samuels 1983 : 30).
\end{abstract}

\title{
Conclusion
}

Les critiques de l'œuvre de l'écrivain remarquent à juste titre le caractère aliénant de la non-acceptation de soi qui marque le début de la carrière académique et littéraire de John Edgar Wideman. Pourtant, en imaginant des identités exclusives et essentielles, c'est-àdire en entérinant l'existence de groupes sociaux sur la base d'une identité, ethnique ou raciale, définie une bonne fois pour toute, la critique afrocentriste substitue à l'impératif critique, un impératif idéologique. Car, si les représentations qui pèsent sur l'identité noire aux Etats-Unis sont définies en référence à une double altérité « du dehors » et « du dedans » (Formoso 2001 : 21), le corpus de catégories du « Je »/《 nous » et du « eux » auxquels l'écrivain a recours témoigne du dynamisme et de la variabilité des relations d'opposition/identification au fondement des processus sociaux de négociation des identités. Certes, le recentrage de l'écrivain autour de son univers familial et social décrit dans la Trilogie de Homewood participe d'une revendication de soi comme acte de liberté, pour autant sa transcription d'une mémoire ostracisée, celle de son quartier, de ses habitants et de sa famille, la restitue au rapport dialectique qui la lie à la société globale. Autrement dit, l'écrivain qui revendique « toute une famille de nègres à Pittsburgh $»^{28}$ force l'action nouvelle de se « remémorer ensemble » et s'affranchit des représentations manichéennes de la société américaine confortées par les « formules narratives canoniques à travers lesquelles les Américains acceptent d'appréhender la vie des Noirs » (Wideman

28 « D'après ce que je pouvais en dire, il n'y avait qu'une alternative : l'un ou l'autre. Riche ou pauvre. Blanc ou noir. Gagner ou perdre. J'ai choisi de quel côté je voulais être quand les saints viendraient pour le Jugement dernier. Qui étaient ces saints, ces souverains de la terre, c'était évident. Mon esprit était divisé par des oppositions, par des catégories s'excluant mutuellement. Manichéisme, comme disait Franz Fanon. Pour réussir dans le monde de l'Homme, il fallait devenir comme l'Homme et l'Homme ne revendiquait sûrement pas toute une famille de nègres à Pittsburgh » (Wideman 1992 [1984] : 46-47). 
$1996: 22)^{29}$. Tout comme, il récuse l'injonction qui lui est faite d'oublier son passé et de se concentrer sur le destin promis par sa réussite académique ${ }^{30}$. Une injonction propre à satisfaire le mythe américain, qui se nourrit de l'amnésie collective et que Toni Morrison décrit en ces termes : " Nous vivons dans un pays où le passé est effacé en permanence et où l'Amérique est représentée comme l'avenir innocent dans lequel des immigrants peuvent arriver et tout recommencer, où les comptes sont constamment remis à zéro » (cité par Gilroy 2003 : 290). Un mythe où l'individu, fût-il noir et né dans un ghetto, est responsable de ses échecs et où celui qui réussit est la preuve vivante de la responsabilité du premier.

Ainsi, l'œuvre de Wideman saisit le mouvement d'élaboration continue d'une mémoire collective américaine - fût-elle négative - et « l'interlocution » de ses différentes mémoires, non plus pensées comme antagoniques, mais dans leur complémentarité. Ce processus exprimant les tensions, les conflits et les enjeux de pouvoir qui transcendent les rapports sociaux.

Par conséquent, l'émergence de la figure de l'écrivain noir signifie, pour les Noirs aux Etats-Unis, la capacité nouvelle d'utiliser la force de l'écrit dans l'espace public, dans l'espace politique. De fait, son existence seule est le signe d'une libération possible, de la subversion d'un privilège de classe et de « race », sur lequel la bourgeoisie (qui n'est plus exclusivement blanche) et la société blanche (qui représente encore l'ordre dominant) a assis et continue d'asseoir une part de son pouvoir ${ }^{31}$. La condition de cette libération impose que ses qualités subversives ne soient pas amoindries par des représentations qui cantonnent la figure de l'écrivain dans une catégorie structurellement dominée. Car, dans une société fortement inégalitaire et «d'insécurité sociale » (Castel 2003), comme aux Etats-Unis, les idéologies d'exclusion sont génératrices de subordination symbolique, mais aussi d'identité. En influençant directement les comportements des acteurs sociaux, elles tendent à assurer un contrôle total sur l'action et sur ses représentations : en contribuant à l'émergence des identités, ces idéologies d'exclusion assurent la pérennité des rapports de force propres au système qui les produit.

J'ai voulu montrer, dans cet article, que si l'écrivain Wideman inscrivait sa démarche littéraire dans un rapport à l'Afrique envisagée pour ses potentialités imaginatives et disruptives, la critique afrocentriste en dessinant l'image d'un écrivain « converti » et en construisant des identités exclusives limitait, le plus souvent, la portée politique et

29 « (...) de l'ignorance à l'éducation, de l'absence de qualification au statut professionnel, du gangster méprisé au porte-parole éclairé (...) La formule du récit d'esclave nouvelle manière se vend parce qu'elle est simple; elle accepte et préserve les catégories du statu quo (comme noir/blanc, par exemple); elle décrit des individus qui traversent des frontières, pas des groupes; elle conforte et rassure les privilégiés tout en offrant une lueur d'espoir aux exclus du pouvoir. Ces récits nous disent que, bien que l'ordre établi rende possible les horreurs des plantations, des ghettos et des prisons, il offre aussi la possibilité d'y échapper. Cet ordre n'est donc pas tout à fait condamnable. Personne n'est totalement coupable, et les opprimés (esclaves, prisonniers, habitants du ghetto) ne sont pas totalement innocents » (Wideman $1996: 22-23$ ).

30 «A Penn Palestre, je suis devenu un meilleur joueur, mais j'ai payé un prix exorbitant pour ça et d'autres affinages culturels. Professeurs, entraîneurs, presque tous les gens importants dans l'environnement blanc de l'université me pressaient d'enterrer mon passé. J'ai appris à trop présumer de qui j'étais, de ce que j'allais devenir. Je vivais pour le jour où je pourrais regarder en arrière, regarder de haut Reds et tout le monde au parc Mellon, à Homewood » (Wideman 1992 [1984] : 325).

31 Cf. Jonathan Kozol, 2005. The Shame of the Nation, The restoration of apartheid, Schooling in America. New York : Crown Publisher. 
les significations sociologiques et anthropologiques de sa littérature. Ici, l'afrocentrisme devient l'instrument ambigu d'une démarche de (re)politisation d'une identité dominée qui, en dernière instance, contribue à la reproduction du devenir minoritaire des Noirs américains. Reste que l'œuvre de John Edgar Wideman de par son foisonnement, sa densité et sa complexité, transcende ces catégories critiques où l'écrivain ne se laisse jamais enfermer. "In fact, one of the reasons I write is to feel free, and that's a different impulse than the impulse to be a member of any particular club, to be a member of any particular group, category, genre, school, gang, clan, hood " (Wideman, in Monville De Cecco 2005).

\section{Références citées}

ANDRADE, Heather Russell, 2006. « Race, Representation, and Intersubjectivity in the Works of John Edgar Wideman ", Critical Essays on John Edgar Wideman, Bonnie TuSmith et Keith E. Byerman (éds), pp. $43-56$.

Asante, Molefi Kete, 2003. L'Afrocentricité. Paris : Menaibuc.

Asante, Molefi Kete et Ama Mazama (éds), 2005. Encyclopedia of Black Studies. Thousand Oaks, Londres, New Dehli : Sage Publications.

Bonetti, Kay, 1998 [1985]. « Interview with John Edgar Wideman », in Bonnie TuSmith (éd.). Conversations with John Edgar Wideman. Jackson : University press of Mississippi, pp. 42-61.

BonNIOL, Jean-Luc,

1992. La couleur comme maléfice. Paris : Albin Michel.

2001. Paradoxes du Métissage, sous la direction de Jean-Luc Bonniol, éditions du CTHS.

Bourdieu, Pierre,

1982. Ce que parler veut dire. Paris : Fayard.

1992. Les règles de l'art, Genèse et structure du champ littéraire. Paris : Seuil.

2004. Esquisse pour une auto-analyse. Paris : Raison d'agir.

Bourgois, Philippe, 2001. En quête de respect, Le crack à New York. Paris : Seuil.

Byerman, Keith E., 2006. «Queering Blackness, Race and Sexual Identity in A Glance Away and Hurry Home », in Bonnie TuSmith and Keith E. Byerman (éds), Critical Essays on John Edgar Wideman, Knoxville : The University of Tennessee Press, pp. 93-105.

Castel, Robert, 2003. L'insécurité sociale : Qu'est-ce qu'être protégé ? Paris : Seuil.

Cesaire, Aimé, 1994. La Poésie. Paris : Seuil.

Coleman, James W., 1989. Blackness and Modernism. Jackson et Londres : The University Press of Mississippi.

Conyers, Jr. James L. (éd.), 2003. Afrocentricity and the Academy, Essays on Theory and Practice. Jefferson, Caroline du Nord et Londres : McFarland \& Company, Inc., Publishers.

ERIBON, Didier, 1999. Réflexions sur la question gay. Paris : Fayard.

Eyerman, Ron, 2001. Cultural trauma, Slavery and the formation of African American identity. Cambridge University Press.

FANON, Franz,

1952. Peau noire, masques blancs. Paris : Seuil.

1961. Les damnés de la terre. Paris : Cahiers libres (27-28), François Maspero éditeur. 
Formoso, Bernard, 2001. «L'ethnie en question, débat sur l'identité », Ethnologie, Segalen (éd.), Armand Collin.

Fox-Genovese, Elizabeth, 1988. Within the Plantation Household, Black \& White Women in the Old South, series on Gender and American Culture. Chapel Hill : University of North Carolina Press.

Franklin, John Hope et Alfred A. Moss, Jr., 2005. From Slavery to Freedom. New York : Alfred A. Knopf.

Gilroy, Paul, 2003 [1993]. L'Atlantique noir. Modernité et double conscience. Editions Kargo (en particulier le chapitre VI où l'auteur se livre à un examen critique de l'Afrocentrisme).

Ginzburg Migliorino, Ellen, 1994. La Marcia Immobile. Storia dei neri americani dal 1770 al 1970. Milan : Selene Edizioni, 1994, le premier chapitre.

Goodman, Joseph. « Cecil Could Run - But he couldn’t Hide », The New York Times, 19 avril 1970.

Goody, Jack, 1979. La Raison graphique. La domestication de la pensée sauvage. Paris : Editions de Minuit, Le sens commun.

Hine, Darlene C., « Female Slave Resistance : The Economics of Sex », Western Journal of Black Studies, 1979 (3), pp. 123-127.

JANIFER, Raymond E., 1997. From Oxford to Homewood: The Long Journey Home of an African-American Rhodes Scholar. Accessible sur internet à l'adresse : http://webspace.ship.edu/rejani/Oth.htm

Kozol, Jonathan, 2005. The Shame of the Nation : The Restoration of Apartheid Schooling in America. New York : Crown Publisher.

Martel, Frédéric, 2006. De la culture en Amérique. Paris : Gallimard.

Mazama, Ama (Cérol, Marie-José Cérol), 2003. L'Impératif Afrocentrique. Paris : Menaibuc.

Mbalia, Doreatha D., 1995. John Edgar Wideman, Reclaiming the African Personality. Selinsgrove : Susquehanna University Press ; Londres et Toronto : Associated University Presses.

MinTZ, Sidney W., 1971. «Une culture à retrouver ou à créer », L’Homme, 11 (3).

Monville De Cecco, Bénédicte, 2005. Entretien avec John Edgar Wideman, New York : avril 2005. Non publié.

O’Brien, John, 1972. «John Wideman », in Conversations with John Edgar Wideman, Bonnie TuSmith (éd.). Jackson : University Press of Mississippi, pp. 5-13.

Raynal, Patrick, « Le Chant du ghetto », Le Monde, 6 mars 1992.

Rerolle, Raphaëlle, « Black dynamite », Le Monde, 15 décembre 2000.

Rosen, Judith, 1998 [1989]. « John Edgar Wideman », in Conversations with John Edgar Wideman, Bonnie TuSmith (éd.). Jackson : University Press of Mississippi, pp. 81-86.

Roskolenko, Henry, « Junkie’s Homecominf », The New York Times, 10 septembre 1967.

Rowell, Charles H., 1998 [1989]. « An interview with John Edgar Wideman », in Conversations with John Edgar Wideman, Bonnie TuSmith (ed.). Jackson : University Press of Mississippi, pp. 86-104.

SAmuels, Wilfred, D, 1998 [1983]. « Going Home : A conversation with John Edgar Wideman », in Conversations with John Edgar Wideman, Bonnie TuSmith (éd.). Jackson : University Press of Mississippi, pp. 14-31.

Shalit, Gene, 1998 [1963]. « The Astonishing John Edgar Wideman », in Conversations with John Edgar Wideman, Bonnie TuSmith (éd.). Jackson : University Press of Mississippi, pp. 1-5.

TuSmith, Bonnie (éd.), 1998. Conversations with John Edgar Wideman. Jackson : University Press of Mississippi.

TuSmith, Bonnie et Keith E. Byerman (éds), 2006. Critical Essais on John Edgar Wideman. Knoxville : The University of Tennessee Press.

Wacquant, Loic, 2005. Race as a Civic Felony. UNESCO et Oxford : Blackwell Publishing Ltd. 


\section{Bénédicte Monville De Cecco}

WIDEMAN, John E.,

1967. A Glance Away. New York : Hardcourt, Brace \& World.

1970. Hurry Home. New York : Harcourt, Brace \& World.

1973. The Lynchers. New York : Henry Holt \& Co.

2002. (éd.) My Soul has Grown Deep, Classics of Early African-American Literature. New York: Ballantine Books.

1978. « Stomping the Blues : Rituals in Black Music and Speech », American Poetry Review (7), pp. $42-45$.

1992 [1984]. Suis-je le gardien de mon frère? Paris : Jacques Bertoin.

1996. «Introduction », in Mumia Abu-Jamal, En direct du couloir de la mort. Paris : La Découverte.

1996 [1990]. L'incendie de Philadelphie. Paris : Gallimard.

1998 [1996]. Le massacre du bétail. Paris : Gallimard.

2004 [1981]. Damballah. Paris : Gallimard.

2006 [1981]. Où se cacher. Paris : Gallimard.

2008 [1983]. Le rocking-chair qui bat la mesure. Paris : Gallimard.

2008. Fanon. Boston, New York : Houghton Mifflin Company. 